\title{
A comparative validation of concepts for collision algorithms for stochastic particle tracking
}

\author{
Philipp Pischke ${ }^{\mathrm{a}, *}$, Reinhold Kneer ${ }^{\mathrm{a}}$, David P. Schmidt ${ }^{\mathrm{b}}$ \\ ${ }^{a}$ Institute of Heat and Mass Transfer \\ RWTH Aachen University, Germany \\ ${ }^{b}$ Mechanical and Industrial Engineering Department \\ University of Massachusetts, Amherst
}

\begin{abstract}
Long-standing concerns about the accuracy of stochastic particle collision algorithms in Monte Carlo simulations have given rise to hybrid collision algorithms, which incorporate ideas from deterministic collision calculations in order to improve the realism of the collision calculation. Some hybrid collision algorithms have spread across commercial and research computational fluid dynamics codes without prior validation. The current work focuses on the predicted incidence of collision, using mathematical analyses and computational tests to validate the stochastic and hybrid concepts. Employing simplified cases permits direct mathematical evaluation of the accuracy of the candidate algorithms. Results indicate that hybrid collision algorithms fail if the predicted collision probability does not scale proportionally to the collision cross-section, relative velocity, number of computational particles and computational time-step. Based on these findings, a general guideline for proper hybridization of deterministic collision algorithms is given. Following these guidelines, hybrid collision algorithms are as accurate as stochastic collision algorithms, with some specific benefits and disadvantages.
\end{abstract}

Keywords:

Collision algorithms; stochastic parcel method; particle-laden flows; validation

\section{Introduction}

Droplet collisions are thought to be very frequent in dense sprays [12], affecting particle size through coalescence and the production of daughter particles [4, 22]. Droplet collisions also exchange momentum between particles and dissipate kinetic energy, since collision outcomes are highly inelastic [17, 21]. In some kinds of atomizers, such as rocket nozzles, sprays are designed to impinge in order to mix fuel and oxidizer through collision. For dense sprays and especially for spray-spray impingement, the collision modeling process should be accurate and reliable.

Unfortunately, the literature does not currently offer a clear picture of how collision modeling should proceed, especially in the context of Lagrangian particle tracking. Lagrangian particle tracking offers a convenient framework for simulating poly-disperse sprays and is particularly popular in applied spray research and industry due to its low cost. Lagrangian particle tracking is capable of representing the highly multi-dimensional spray equation [27] where velocity may not be a single-valued function of space and time. Due to the large number of particles present in a typical spray, however,

${ }^{*}$ Corresponding author

Email addresses: pischke@wsa.rwth-aachen.de (Philipp Pischke), kneer@wsa.rwth-aachen.de (Reinhold Kneer), schmidt@ecs.umass.edu (David P. Schmidt) 
Lagrangian particle tracking generally uses statistical sampling so that only a subset of actual particles are tracked. This sampling makes collision calculations a much more complicated endeavor.

There are two fundamental parts to the collision calculation: the incidence of collision and the outcomes of collision. The latter topic represents the complex physics of collision and has seen numerous improvements from experiments and detailed simulation $[2,4,6,14,16,18,23]$. The former topic, however, has proven more problematic: the mathematics of these Monte-Carlo simulations are often counter-intuitive, resulting in some confusion over what methods are appropriate for calculating the incidence of particle collision.

When only a sample of the actual particles are tracked, and since the representative tracked particles have varying statistical weights [27], the collisions must be calculated stochastically. In a given computational volume, the probability of collision is based on the variance in velocity of the particle population, the number density of the particles, and the particle sizes. O'Rourke produced a seminal work that established how a stochastic algorithm could be constructed. His model was inspired by analogous work in gas dynamics, where researchers were concerned with molecular collisions [17].

MacInness and Bracco [12] provided an early challenge to O'Rourke's algorithm, by comparing it to spray computations performed with direct particle tracking. They reported that the stochastic method underestimated collision and coalescence rates by a factor of approximately 2 , which has not been confirmed by other authors. Since then, others have taken issue with O'Rourke's algorithm as well, offering various criticisms and improvements.

Schmidt and Rutland [24] found strong evidence to support the correctness of O'Rourke's work. Though there was evidence of high statistical scatter, they noted convergence and consistency of the incidence of collision calculations when sufficient mesh resolution was employed. As a general shortcoming of mesh-based collision algorithms, Schmidt and Rutland [24] noted the grid dependency that typically occurred in collision calculations due to under-resolution. They suggested that collision computations should use a separate collision mesh and a faster "No-Time-Counter" algorithm. Most importantly, this work tested the incidence of collision directly against analytical solutions by assuming simplified collision outcomes. This approach removed confounding complications created by complex physics and allowed high-fidelity testing of the predictions of collision incidence. Later work generalized the proposed algorithm and provided further mathematical support and validation [10]. Other algorithms make use of moving control volumes [13] to avoid mesh artifacts.

In two further studies, remaining errors of stochastic algorithms were identified. These errors originate from the assumption of isotropic dispersions ("voidage errors") and from steep velocity gradients ("gradient errors"). While voidage errors can be addressed by anisotropic refinement of the control volumes [20], gradient errors can be eliminated by reconstructing the velocity gradient in a least square manner and eliminating it's influence on relative velocities [21]. The developed algorithms have been validated following the methodology proposed by Schmidt and Rutland [24].

Despite the strong validation of stochastic collision algorithms, one assertion in the literature is that stochastic methods are flawed because they do not consider whether two computational particles or "parcels" are moving towards each other or moving apart. An early example of this idea appeared in work by Nordin [15], and is also found in Gwon and Ryou [8] and Pawar et al. [19].

Another idea is that there is a certain maximum time for collision or distance between parcels, beyond which collision should be excluded. This concept was employed by Munnannur and Reitz [13], who imposed a mean collision time constraint, and by Abani et al. [1]. In a study of particle collision in icing, Kollár et al. [11] decided that "if the distance 
between two parcels is less than the sum of their radii, they will collide". Several of these criteria were used by Gustavsson and Golovitchev [7].

Direct Numerical Simulations such as Chen et al. [5] that directly track all particles naturally make use of such deterministic logic. It is the decision to track only a statistical subset of particles that has lead to stochastic methods. Algorithms that are partially based on stochastic methods, but utilize collision constraints based on deterministic ideas, are "hybrid" collision algorithms, which are becoming more widely embraced by the computational fluid dynamics community. For example, they appear in the widely used OpenFOAM ${ }^{\circledR}[30]$ software and in the AVL Fire ${ }^{\circledR}$ code [3]. Though many of these ideas have an intuitive appeal, they should be rigorously validated. In this paper, we offer a mathematical analysis of their validity. In a subsequent section, we employ numerical tests on simplified problems that permits unambiguous assessment of the correctness of results. As established in previous publications [24], the testing methods will focus on the validation of the incidence of collision calculation, since collision outcomes are a separate matter.

\section{Review of algorithms}

Due to the large number of different collision algorithms available, this work will focus on three candidate algorithms. This section first presents a deterministic algorithm for the case where particle collisions are simulated based on their trajectories, which is then hybridized for Monte Carlo simulations by proper scaling of the collision probability. Next, we review O'Rourke's stochastic method [17], the seminal point from which other stochastic collision algorithms have been derived. Finally, Nordin's hybrid algorithm is presented [15], which combines stochastic collision calculations with deterministic features, and has been chosen due to its popularity in computational fluid dynamics codes.

\subsection{Deterministic algorithm}

Deterministic approaches check the trajectories of every two particles $i$ and $j$ instead of relying on stochastic means. A formulation for the given collision conditions can be found by searching the minimum distance $\mu_{0}$ between the particle trajectories,

$$
\boldsymbol{X}_{\boldsymbol{i}}=\boldsymbol{x}_{\boldsymbol{i}}+t \boldsymbol{u}_{\boldsymbol{i}}, \quad \boldsymbol{X}_{\boldsymbol{j}}=\boldsymbol{x}_{\boldsymbol{j}}+t \boldsymbol{u}_{\boldsymbol{j}}, \quad \frac{\partial \overbrace{\left\|\boldsymbol{X}_{\boldsymbol{i}}-\boldsymbol{X}_{\boldsymbol{j}}\right\|}^{=\mu}}{\partial t}\left(t_{0}\right)=0
$$

where $t_{0}$ is the instance in time at which particles pass. The solution of the minimization problem leads to

$$
t_{0}=-\frac{\left\langle\boldsymbol{u}_{\boldsymbol{i j}}, \boldsymbol{x}_{\boldsymbol{i j}}\right\rangle}{\left\langle\boldsymbol{u}_{\boldsymbol{i j}}, \boldsymbol{u}_{\boldsymbol{i j}}\right\rangle}
$$

Here, $\boldsymbol{x}_{\boldsymbol{i} \boldsymbol{j}}$ is the position of particle $j$ relative to particle $i$, and $\boldsymbol{u}_{\boldsymbol{i} \boldsymbol{j}}$ the relative velocity. To constrain collision to the present time-step, the time $t_{0}$ until the minimum distance $\mu_{0}$ must remain within the limits of $0 \leq t_{0}<\Delta t$, Fig. 1 . A collision occurs only if the particles pass within the circle of the collision cross-section sigma defined by the two particle radii, $r_{i}$ and $r_{j}$ :

$$
\sigma=\pi\left(r_{i}+r_{j}\right)^{2}
$$

such that

$$
\pi \mu_{0}^{2}<\sigma
$$




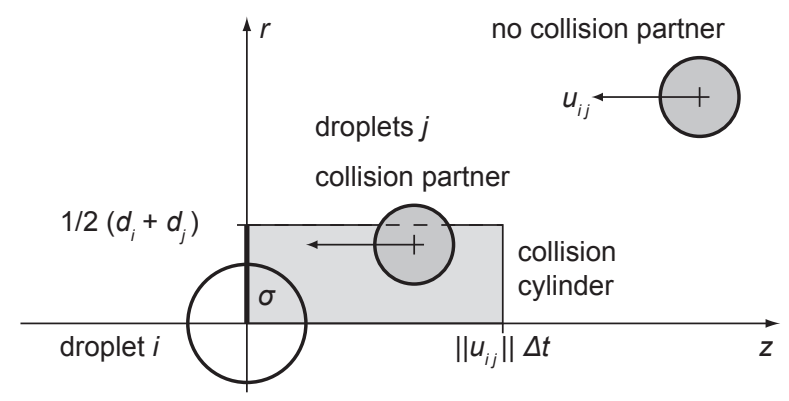

Figure 1: Deterministic logic of direct collision calculations. A collision occurs if two particle pass within the circle of the collision cross section within the next time-step. The collision cylinder formed by the displacement and collision cross-section is the basis to stochastic collision calculations.

Then, one collision of particle $i$ and $j$ is modeled.

For the typical case where stochastic parcels are used, the deterministic algorithm may still be part of the overall Monte-Carlo approach, although only a stochastically chosen subset of particles are tracked, each representing $q$ particles of the entire population (where $q$ is not necessarily an integer). To transfer the deterministic approach to stochastic parcel methods, the number of collisions must be scaled by the number of particles per parcel $i$ and $j$, i.e. by the product $q_{i} q_{j}$. This can be achieved by scaling either the collision probability, or the number of collisions to be modeled, or both.

From the point of view of parcel $i$, the available cross-section in (4) is scaled by the number of particles $q_{j}$ represented by parcel $j$, as multiple cross-sections are available:

$$
\pi \mu_{0}^{2}<\sigma q_{j}
$$

If condition (5) is met, then $q_{i}$ collisions are modeled. In the case of stochastic parcel simulations where the number of particles $q_{i}$ and $q_{j}$ are not equal to unity, the approach scales the collision probability by $q_{j}$, and the number of collisions per incident by $q_{i}$, which leads to proper scaling of the overall number of collisions. This approach will be designated as the "weighted deterministic" algorithm, and is an example of proper hybridization of deterministic algorithms. In the limit of each parcel representing one particle, this approach reduces to direct tracking of particles.

\subsection{O'Rourke's algorithm [17]}

When using parcels, the exact locations of all particles are not known. Instead, the collision algorithm must use the known locations of the parcels for computing collisions stochastically. The derivation begins, however, with consideration of two particles.

From geometric considerations, two particles collide within the forecasted time-step $\Delta t$ if the centers of the two particles pass within a circle defined by the collision cross section. In a cylindrical coordinate system aligned with the relative velocity, these conditions form a collision cylinder of volume

$$
V_{\text {coll }}=\sigma\left\|\boldsymbol{u}_{i j}\right\| \Delta t
$$

as shown in Fig. 1 above. While direct numerical simulations find collisions by checking the prior criteria deterministically, O'Rourke's algorithm begins by calculating the probability that a single particle from one parcel will impact any particles from another parcel within a given control volume. 


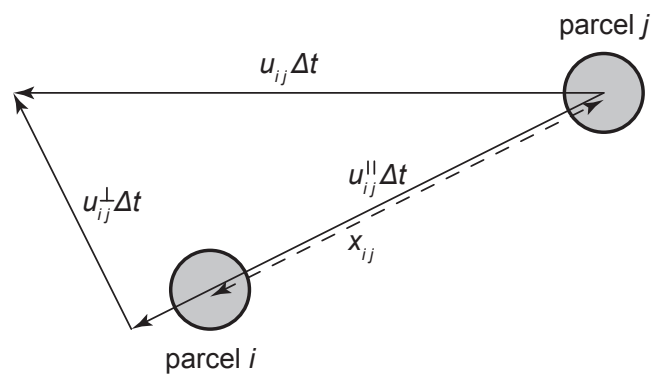

Figure 2: Deterministic logic of Nordin's hybrid algorithm. The symbol $u_{i j}$ represents the relative velocity. In Nordin's hybrid algorithm, collisions occur if $u_{i j}^{\|} \Delta t>\left\|\boldsymbol{x}_{\boldsymbol{i}}-\boldsymbol{x}_{\boldsymbol{j}}\right\|$, which is a non-physical condition, as the parcels in this example adhere to this condition yet do not collide.

As particles are assumed to spread homogenously across the control volume, the collision probability $P_{i j}$ of parcel $i$ colliding with parcel $j$ is equal to the probability of presence within the collision cylinder, which is the volume ratio of the collision cylinder and the control volume:

$$
P_{i j}=q_{j} \frac{V_{\text {coll }}}{V}=q_{j} \frac{\sigma\left\|\boldsymbol{u}_{i j}\right\| \Delta t}{V}
$$

To determine if a collision occurs or not, stochastic collision algorithms employ a simple acceptance/rejection scheme. If some uniform random number is smaller than the collision probability $P_{i j}$, then $q_{i}$ collisions must be modeled. Otherwise, no collision occurs.

O'Rourke intended to generalize his algorithm for the case where multiple collisions per time-step could occur, i.e. for $P_{i j}>1$. In this scenario, a single particle from parcel $i$ collides with multiple particles from another parcel $j$. To model this, O'Rourke samples the number of collisions from a Poisson distribution. Since the Poisson distribution is for modeling multiple independent events, its application to particle collisions, where the outcomes significantly change particle sizes and relative velocities, is questionable; a linear approach to multiple collisions is presented in Schmidt and Rutland [24].

\subsection{Nordin's hybrid algorithm [15]}

Hybrid collision algorithms generally consist of two steps. In the deterministic step, potential collision partners are selected based on their trajectories. In the stochastic step, the collision probability is determined by particle distance and displacement. For Nordin's hybrid algorithm [15], two conditions are given to find potential collision partners:

1. "In order for two parcels to collide they must be moving towards each other." The velocity magnitude in the direction of the distance vector is found by

$$
u_{i j}^{\|}=-\left(\boldsymbol{u}_{\boldsymbol{i}}-\boldsymbol{u}_{\boldsymbol{j}}\right) \frac{\left(\boldsymbol{x}_{\boldsymbol{i}}-\boldsymbol{x}_{\boldsymbol{j}}\right)}{\left\|\boldsymbol{x}_{\boldsymbol{i}}-\boldsymbol{x}_{\boldsymbol{j}}\right\|}>0
$$

where the condition $u_{i j}^{\|}>0$ ensures that only parcels may collide that are actually approaching each other.

2. "The parcels' relative displacement must be larger than the distance between them." This constraint is intended to ensure that parcels are close enough that their motion could actually bring them together. The original formulation for this condition is given by

$$
u_{i j}^{\|} \Delta t>\left\|\boldsymbol{x}_{\boldsymbol{i}}-\boldsymbol{x}_{\boldsymbol{j}}\right\|-\left(r_{i}+r_{j}\right)
$$


This mathematical condition is wrong, as it neglects the velocity component $u_{i j}^{\perp}$ that is orthogonal to $\boldsymbol{x}_{\boldsymbol{i j}}$. If this component were very large, two parcel may pass although (9) holds, as shown in Fig. 2.

If the particle radius is neglected over the parcel distance, (8) and (9) yield

$$
t_{0}=-\frac{\left\langle\boldsymbol{x}_{\boldsymbol{i j}}, \boldsymbol{x}_{\boldsymbol{i j}}\right\rangle}{\left\langle\boldsymbol{x}_{\boldsymbol{i j}}, \boldsymbol{u}_{\boldsymbol{i j}}\right\rangle}
$$

which is a different equation when compared to (2), i.e. the condition in (9) does not put any physically reasonable constraint on parcel trajectories.

For each pair of parcels that meet the given collision conditions in (8) and (9), the collision probability is calculated, and collisions are triggered stochastically by an acceptance/rejection scheme. For Nordin's hybrid algorithm [15], the collision probability is defined by the closest distance of the trajectories $\left\|\mu_{0}\right\|$. It is found by solution of

$$
\boldsymbol{X}_{\boldsymbol{i}}=\boldsymbol{x}_{\boldsymbol{i}}+\alpha \boldsymbol{u}_{\boldsymbol{i}}, \quad \boldsymbol{X}_{\boldsymbol{j}}=\boldsymbol{x}_{\boldsymbol{j}}+\beta \boldsymbol{u}_{\boldsymbol{j}}, \quad \frac{\partial \overbrace{\left\|\boldsymbol{X}_{\boldsymbol{i}}-\boldsymbol{X}_{\boldsymbol{j}}\right\|}^{=\mu}}{\partial \alpha, \beta}\left(\alpha_{0}, \beta_{0}\right)=0
$$

where $\alpha$ and $\beta$ are the time of motion. Although similar to (1), the oddity of this formulation is that both parcels move in different temporal dimensions given by $\alpha \neq \beta$, i.e. $\|\mu\|$ is the distance between two particles at different instances in time. A consistent formulation would set $\alpha=\beta=t$, leading back to (1). The introduction of independent time-scales leads to many numerical and physical issues, which put Nordin's hybrid algorithm in question entirely.

For the general case of $\boldsymbol{u}_{\boldsymbol{i}} \nVdash \boldsymbol{u}_{\boldsymbol{j}}$ the minimization of (11) is found by solving the matrix equation

$$
\left(\begin{array}{ccc}
\mid & \mid & \mid \\
\boldsymbol{u}_{\boldsymbol{i}} & -\boldsymbol{u}_{\boldsymbol{j}} & \boldsymbol{n}_{\boldsymbol{i j}} \\
\mid & \mid & \mid
\end{array}\right)\left(\begin{array}{l}
\alpha_{0} \\
\beta_{0} \\
\mu_{0}
\end{array}\right)=\boldsymbol{x}_{\boldsymbol{j}}-\boldsymbol{x}_{\boldsymbol{i}}
$$

where $\boldsymbol{n}_{\boldsymbol{i} \boldsymbol{j}}$ is unit-normal on $\boldsymbol{u}_{\boldsymbol{i}}$ and $\boldsymbol{u}_{\boldsymbol{j}}$ as given by the cross product

$$
n_{i j}=\frac{u_{i} \times u_{j}}{\left\|u_{i} \times u_{j}\right\|}
$$

and $\left\|\mu_{0}\right\|$ is the minimum distance between the parcels if $\alpha_{0}$ and $\beta_{0}$ were independent time scales, as noted before. For the special case of $\boldsymbol{u}_{\boldsymbol{i}} \| \boldsymbol{u}_{\boldsymbol{j}}$ or $\boldsymbol{u}_{\boldsymbol{i}}=0$ or $\boldsymbol{u}_{\boldsymbol{j}}=0$, there is no solution to $\alpha_{0}$ and $\beta_{0}$ as the rank of the matrix in (12) is smaller than 3. Hence, the very simple cases of one particle moving and all other particles non-moving, or particles moving parallelly, cannot be described, i.e. the algorithm is not generalizable.

Another requirement for any collision algorithm is independence of the frame of reference. If $\boldsymbol{v}$ is the velocity of the reference system, i.e. let $\boldsymbol{u}_{\boldsymbol{i}}=\boldsymbol{u}_{\boldsymbol{i}}^{*}+\boldsymbol{v}$ and $\boldsymbol{u}_{\boldsymbol{j}}=\boldsymbol{u}_{\boldsymbol{j}}^{*}+\boldsymbol{v}$, the minimum distance $\left\|\mu_{0}\right\|$ is a function of $\boldsymbol{v}$, i.e. the response of the algorithm is not independent of the frame of reference. Therefore, even if the algorithm were correct for one specific frame of reference, it is incorrect for any other. Collision probability must be based strictly on relative velocity, not absolute velocity, in order to provide reference frame independence.

After solution of (12) and the determination of $\alpha_{0}, \beta_{0}$ and $\mu_{0}$, the collision probability in Nordin's algorithm is proposed to decay with distance of the trajectories [15],

$$
P_{i j}=\underbrace{\left(\frac{r_{i}+r_{j}}{\max \left(r_{i}+r_{j},\left\|\mu_{0}\right\|\right)}\right)^{C_{1}}}_{=K_{\mathrm{s}}} \underbrace{\exp \left(-C_{2} \frac{\left\|\alpha_{0}-\beta_{0}\right\|}{\Delta t}\right)}_{=K_{\mathrm{t}}}
$$


where $K_{\mathrm{s}}$ and $K_{\mathrm{t}}$ are spatial and temporal kernels to restrict collision probabilities. The formulation of the denominator $\max \left(r_{i}+r_{j},\left\|\mu_{0}\right\|\right)$ is an ad-hoc fix to avoid near-infinite collision probabilities for close-by trajectories. Model constants are given by $C_{1}=1$ and $C_{2}=0$ to 0.3 .

Although qualitatively reasonable, two scaling problems may be identified in the formulation of the collision probability: from deterministic considerations, the collision probability scales by the collision cross-section $\sigma$, i.e. by $\left(r_{i}+r_{j}\right)^{2}$, hence only a value of $C_{1}=2$ can lead to correct results. Also, any consideration of the number of particles represented by the parcels is missing. If the number of parcels is reduced, mean distances between parcels will grow, leading to reduced collision probabilities. This must be compensated for by adequate scaling of the collision probability, as shown in the derivation of the weighted deterministic algorithm.

Besides inadequate scaling, the choice of the exponent $C_{1}$ imposes another problem of the spatial kernel: One requirement of any kernel is that its integral is finite; integrating the probability of collision should result in a finite expected value even in an infinite domain. For stochastic parcel simulations, a finite box kernel is given implicitly by the control volume. However, the kernel $K_{\mathrm{s}}$ introduced explicitly in (14) integrates to infinity for $C_{1}=1$ :

$$
\int_{0}^{\infty}\left(\frac{r_{i}+r_{j}}{\max \left(r_{i}+r_{j}, \mu_{0}\right)}\right)^{C_{1}} d \mu_{0}=\int_{0}^{r_{i}+r_{j}}\left(\frac{r_{i}+r_{j}}{r_{i}+r_{j}}\right) d \mu_{0}+\int_{r_{i}+r_{j}}^{\infty}\left(\frac{r_{i}+r_{j}}{\mu_{0}}\right) d \mu_{0}=\left(r_{i}+r_{j}\right)+\infty
$$

Thus, in a quasi-infinite domain with quasi-infinite collision partners, the collision probability of a particle sums up to infinity at any location and point in time. This is an indication that the kernel is not sufficiently diminishing in the far-field. While the above integral is performed in one dimension for clarity, an integral in three-dimensional spherical coordinates would not change the conclusion; the extra factor of $4 \pi \mu_{0}^{2}$ would only increase the weighting of distant particles. For the kernel to converge, the exponent must be chosen such that $C_{1}>3$, which again leads to inproper scaling with the collision cross-section. Pischke et al. have demonstrated Gaussian kernels that have not shown these issues [20], but led to proper scaling of the collision cross-section.

Finally, from a practical point of view, the Nordin hybrid algorithm depends upon four numerical parameters, i.e. the number of parcels, the numerical time-step, and two model constants, while most stochastic collision algorithms depend on two parameters only, i.e. the number of parcels and the numerical time-step. As stated by Nordin [15], the present collision is mesh-independent, as it is not based on any mesh information. However, this independence is bought by introduction of two arbitrary model constants, which introduce other numerical dependencies.

\section{Analysis and Tests}

Because the ultimate goal of modeling is to faithfully represent actual phenomena, model testing often focuses on comparison to experimental data. However, in this case, we are testing an algorithm, and thus a numerical method. The focus of the present work is the incidence of collision, which is a mathematical and algorithmic challenge, rather than a problem of fluid dynamics. In the case of collision efficiency models or turbulence-driven collision [26, 28, 29], which are not considered here, complex physics would become part of the incidence calculation.

For comparison of the candidate algorithms, two test cases are introduced. The first test case resembles Beer's law, i.e. the attenuation of light intensity by molecular absorption. The strength of this test case is that Beer's law is undisputed, which allows to compare results against a generally accepted solution. Specific to the Beer's law test case is the 
presence of two populations, a molecule and a photon population, with interaction between, not within the populations. As photons move through the molecule population, the Beer's law test case is capable of showing temporal convergence of photon absorption. The present test case has one population of moving particles, analogous to the photons, and a stationary population, analogous to the molecules.

The second test case has been taken from a previous publication [24], validating the collision rate for a spatially inhomogeneous particle population. Here, interaction occurs within the population. As only the collision rate is predicted, i.e. the number of collisions for one time-step after initializing the population, the collision rate test case cannot show temporal convergence, but spatial convergence and response to the number of parcels for inhomogeneous distributions.

\subsection{Beer's law}

In order to directly test the incidence of collision we use a test problem that is universally accepted as true and is simple enough to admit an analytical solution. By using such a test problem, we can clearly know that any errors are due to the collision incidence algorithm and are not a consequence of other models, such as the collision outcomes. Further, the availability of an analytical solution obviates the need to rely entirely on the comparison of computations to other computations for validation, as in MacInness and Bracco [12] or Pischke et al. [20].

For our chosen test, we will have two groups of particles. The first group is stationary and of uniform size, distributed with a statistically uniform concentration. We refer to this group of particles as the "molecule population." A second group of particles with their own uniform radius value moves with parallel trajectories through the stationary particles, representing the "photon population." Because the members of each group of particles has equal velocities, collisions only occur between the two groups, not within each group. Whenever a photon passes within the circle of the collision cross-section, a collision occurs which results in annihilation of the photon and has no impact on the molecule. This scenario results in a gradual extinction of the photon flux and is governed by Beer's law.

This example, though simplified, actually represents a much more realistic test than is first evident. Collision should be independent of the frame of reference, so we can freely change reference frames to make these two particles have more general velocities, instead of one moving and one fixed. Also, for short times, collision of a general population of poly-disperse particles can be represented as a superposition of many sub-groups colliding. The present analysis represents the collision of two sub-groups, that could represent the more general case where both are in motion by using a shift in reference frame.

Prior to undertaking numerical experiments, we can analyze what O'Rourke's method would predict for this problem using a mathematical analysis. Here, we consider the limit of sufficiently small time-steps that the possibility of O'Rourke's algorithm predicting multiple collisions can be neglected, e.g. $P_{i j}<1$. In the analysis below, we denote the photons with the subscript $i$ and the molecules with the subscript $j$. It is assumed that their motion is aligned with the $x$ axis. The extinction of photons is a consequence of geometry, with the cross-section of the interaction given by $\sigma$ as defined in (3).

Consider a flux of moving photons, $I(x)$, entering a small volume located at $x$ and leaving the volume with an intensity flux $I(x+\Delta x)$. The difference between these two fluxes is the extinction per unit area normal to the flux. This extinction can be expressed using the probability, $P_{i j}$ of a single photon $i$ colliding with a molecule parcel $j$, as introduced in ( 7 ). Using O'Rourke's algorithm, the extinction is equal to the number of expected collisions, i.e. the product of the photon 
flux $I(x)$ and the probability of one collision $P_{i j}$ for all molecules $\sum q_{j}$,

$$
I(x+\Delta x)-I(x)=I(x) \sum_{j} \frac{q_{j}}{V} \sigma \cdot\left\|\boldsymbol{u}_{\boldsymbol{i j}}\right\| \Delta t
$$

We can note that the product of the velocity and a differential time interval gives a differential distance, $\left\|\boldsymbol{u}_{\boldsymbol{i j}}\right\| \Delta t=\Delta x$. Also, the number of molecules $\sum q_{j}$ per volume $V$ is the molecule number density $n$. Thus (16) reduces to

$$
I(x+d x)-I(x)=I(x) n \sigma \Delta x
$$

where $1 / n \sigma$ is the mean free path. If the photons are distributed with an initial intensity flux $I_{0}$ their intensity will be governed by Beer's law:

$$
I=I_{0} \cdot \exp (-n \sigma x)
$$

In the limit of small $\Delta x$, the solution to (17) predicts an exponential decline in the number of photons as they proceed in the $x$-direction as given by (18). Thus, this analysis shows that with sufficiently small time-step, the average prediction of O'Rourke's method is convergent and consistent for this problem. Since the exact answer is recovered by O'Rourke's algorithm in the limit of vanishing $\Delta t$ and $\Delta x$, this analysis also suggests that the exclusions imposed on collision partners by hybrid methods cannot be correct unless they have no effect in the limit of small $\Delta t$ and $\Delta x$.

For the numerical analyses of the algorithms introduced, the photon parcels are tracked over multiple time-steps $\Delta t$ through the domain. The error at location $\mathrm{x}$ is defined by

$$
\text { err }=\frac{I_{\text {num }}(x)}{I_{\text {theo }}(x)}-1
$$

where the indices (theo) and (num) denote the theoretical and numerical solutions, respectively. For each case investigated, 400 independent runs are performed, as any collision calculations underly statistical scatter if parcel positions are random. In previous publications [20,24], the mean absolute error was investigated, which does not allow to distinguish between systemic errors and random fluctuations. In this study, the overall error is found as the root mean square of the run-to-run mean error and its standard deviation,

$$
\overline{\text { err }}=\underbrace{\operatorname{rms}(\overbrace{\text { mean }(\mathrm{err})}^{\text {all runs }})}_{\text {all time-steps }}
$$

and

$$
\widetilde{\text { err }}=\underbrace{\operatorname{rms}(\overbrace{\text { std (err) }}^{\text {all runs }})}_{\text {all time-steps }}
$$

For the mean error, a $95 \%$ confidence interval (CI) will be given. Outside the confidence interval, the mean error is an indicator for systemic errors, whereas the standard deviations indicates run-to-run fluctuations. Inside the confidence interval, mean errors may be a residual of run-to-run fluctuations; the mean error does not allow a conclusion of any systemic convergence behavior.

For finite time-steps $\Delta t$ as given in Fig. 3, the O'Rourke algorithm shows first order convergence $\mathcal{O}(\Delta t)$, as it performs an explicit Eulerian integration of an ordinary differential equation. The hybrid deterministic algorithm does not show 


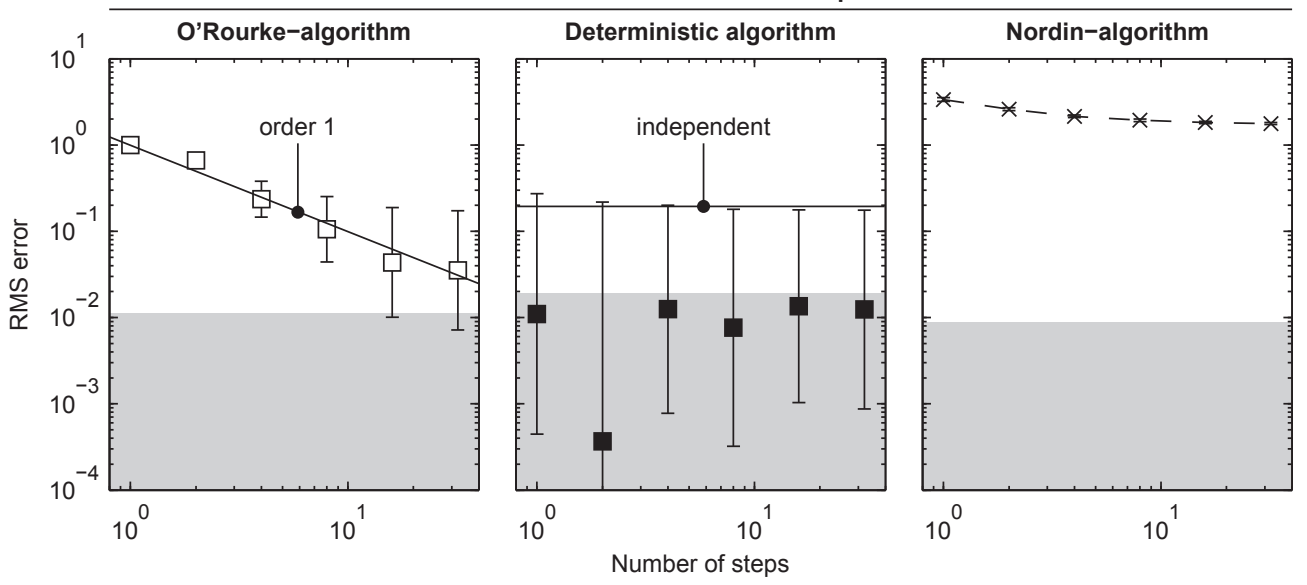

Figure 3: Response of the Beer's law test case to variable numbers of steps. The gray area indicates the $95 \%$ confidence interval (CI) in which the mean error may be residual. The O'Rourke-algorithm converges by $\mathcal{O}(\Delta t)$ as known from other publications [24]. The mean error of the weighted deterministic algorithm is within the confidence interval and thus probably residual; the variance is independent. Nordin's hybrid algorithm fails to converge for increasing and decreasing numbers of steps.

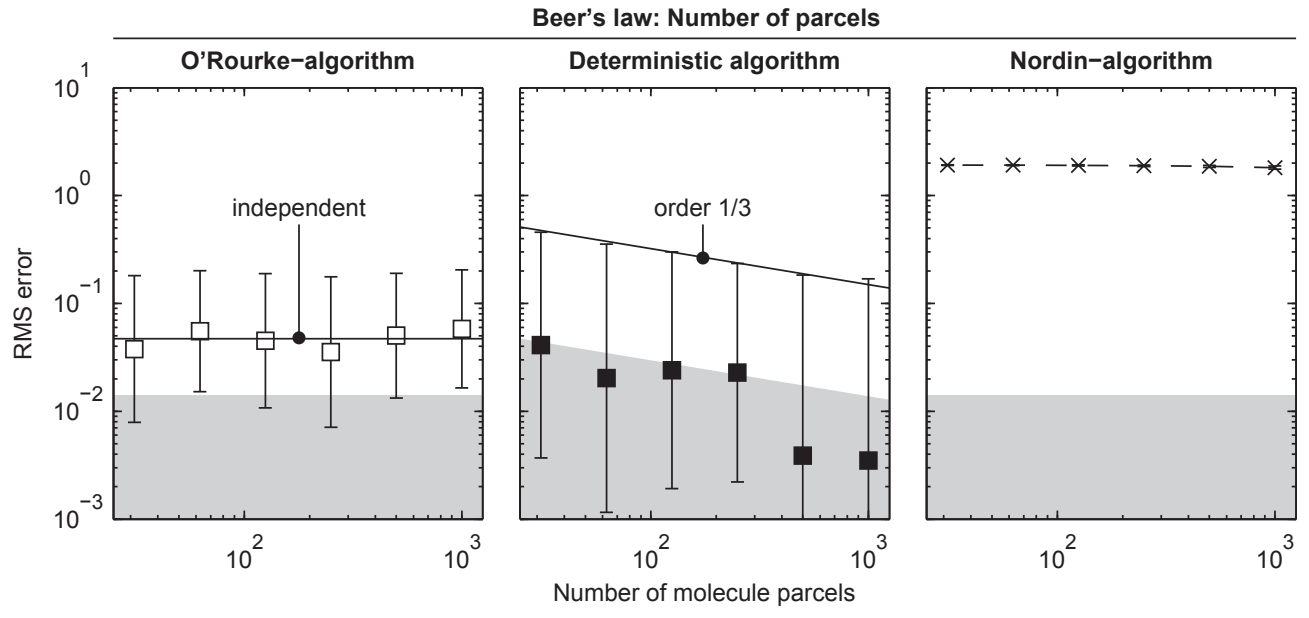

Figure 4: Response of the Beer's law test case to variable numbers of molecule parcels. The gray area indicates the $95 \%$-CI in which the mean error may be residual. The mean errors and variances of the O'Rourke-algorithm are independent of the number of parcel; the remaining error of the O'Rourke-algorithm originates from the limited number of steps as was shown in Fig. 3. The weighted deterministic algorithm shows $\mathcal{O}\left(N_{\mathrm{p}}^{1 / 3}\right)$ convergence of variance, with residual mean errors well within the confidence interval. Nordin's hybrid algorithm fails to converge.

any time-step dependency, and mean errors are well within the confidence interval, i.e. the mean errors are unlikely to be systemic, but rather residual error from an insufficient number of independent runs. Nordin's hybrid algorithm shows significant systemic errors, which do not converge for either large or small step sizes, which is due to the malformed temporal kernel in (14) and (15). 


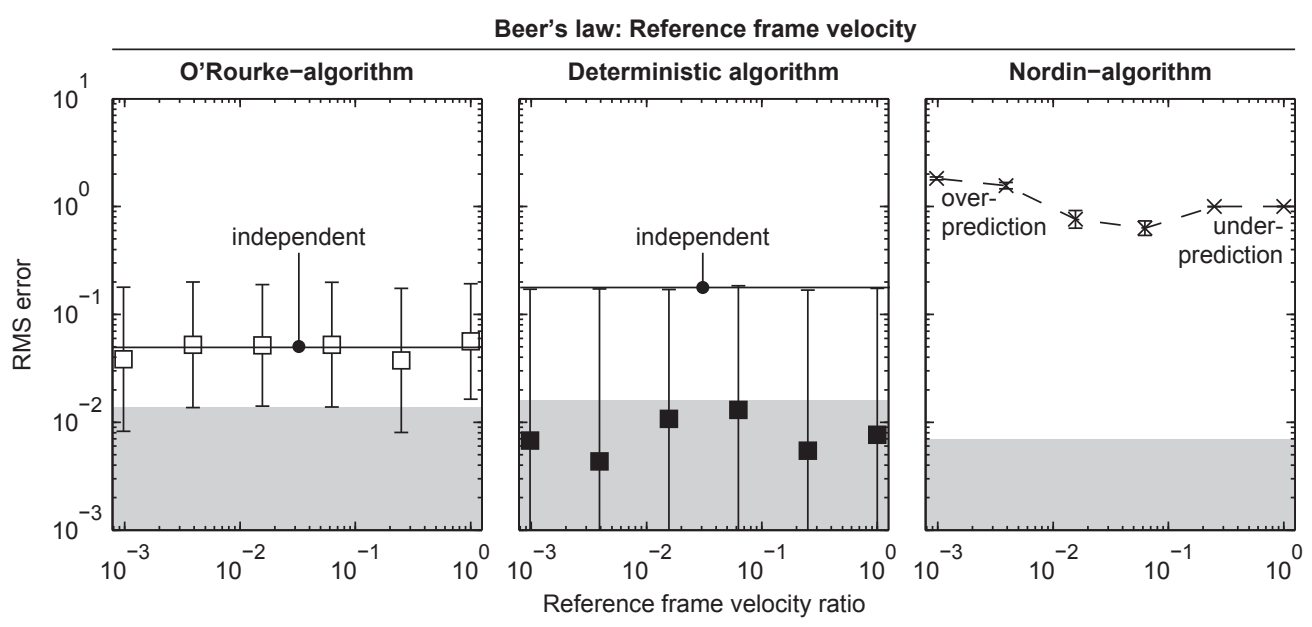

Figure 5: Response of the Beer's law test case to variable reference frame velocity ratios (ref. frame velocity/relative velocity). The gray area indicates the $95 \%$-CI in which the mean error may be residual. The mean errors and variances of the O'Rourke- and deterministic algorithms are independent, which is required behavior; Nordin's hybrid algorithm diverges in both directions, from an over-prediction for small reference frame velocities to an under-prediction for large reference frame velocities (not visible in the figure, as the logarithmic scale shows absolute errors).

In Fig. 4, the response of the algorithms to a variation of the number of parcels $N_{\mathrm{p}}$ is shown, where the overall number of photons and molecules is kept constant by adjusting $q$ proportionally. Contrary to other findings [20, 21, 24, 25], the O'Rourke-algorithm does not show any significant response to the number of parcels of both the mean error and its standard deviation. The reason for the insensitivity is that the collisions between two different populations is investigated, contrary to the named studies, where collisions within one single population were subject to study. The remaining mean error originates from the finite time-step as discussed before, and will converge for vanishing time-steps. In contrast, the weighted deterministic algorithm appears to be exact on average, as mean errors are within the confidence interval. Standard deviations are larger than those provided by O'Rourke's algorithm, which is an indication for increased statistical scatter. Nordin's algorithm shows a significant mean error for small number of parcels and no consistent convergence. The nearly invisible decline of the error does not lead to convergence to the exact solution. On the logarithmic scale, standard deviations are small; in absolute numbers, it is comparable to the other algorithms.

As expected, a change in reference frame velocity influences neither the O'Rourke algorithm nor the weighted deterministic algorithm, Fig. 5. The mean error of the O'Rourke algorithm originates from the limited step size as discussed before, and thus is systemic. The mean error of the hybrid deterministic algorithm falls within the confidence interval and thus is most probably a residual of the finite number of independent runs. As expected from the discussion, Nordin's hybrid algorithm shows a strong dependency on reference frame velocity. Towards slow reference frame velocities, the algorithm shows an over-prediction with an absolute error greater than 1 and growing. Towards high reference frame velocities, the algorithm does not predict any collisions with an absolute error of 1 . In between the over- and under-prediction, there is a reference frame velocity at which the error is vanishing, however, this is not convergence, but coincidence. 


\subsection{Collision rate with variable number densities}

This test is intended to reveal the performance of each method for the case of spatially varying problems. For the collision rate test case, particle number densities are distributed with linearly increasing number densities along the $x$-axis of the domain; at $x=0$, the number density is $n(x=0)=0$. Particle diameters and velocities $u$ in $x$-direction are sampled from uniform random distributions with maximum diameters and velocities of $d_{0}$ and $u_{0}$; the other velocity components are zero. As Nordin's hybrid algorithm cannot handle non-moving particles as discussed for (11), a reference frame velocity $v$ is added in $y$-direction and thus perpendicularly to $u$.

Contrary to the previous test case, where a moving population was tracked through a domain, the collision rate test case evaluates the instantaneous collision rate $\nu=d P / d t$ at one instance in time. For this test case, the error is defined in terms of the theoretical collision rate:

$$
\text { err }=\frac{\nu_{\text {num }}}{\nu_{\text {theo }}}-1
$$

As only one instance in time is observed, there is averaging over multiple steps as in the error definitions for the Beer's law test case in (20) and (21)

$$
\overline{\text { err }}=\overbrace{\operatorname{mean}(\text { err })}^{\text {all runs }}
$$

and

$$
\widetilde{\text { err }}=\overbrace{\text { std }(\text { err })}^{\text {all runs }}
$$

As before, the destinction of mean and standard deviations allows to separate systemic errors from statistical fluctuations.

In the numerical experiment, the collision rate is found by dividing the collision probability by the computational time-step, i.e. $\nu=P / \Delta t$. For O'Rourke's algorithm, Fig. 6 does not indicate any time-step dependencies, as the time-step $\Delta t$ is eliminated from the collision probability in (7). The deterministic algorithm shows a constant systemic error, viz. an under-prediction, which cannot be seen by the logarithmic scale. The error originates from parcels leaving into the void region outside the domain, where no collisions occur. Increased time-steps lead to more potential collisions and thus better statistics, reducing the standard deviation by $\mathcal{O}\left(\Delta t^{1 / 2}\right)$. Nordin's hybrid algorithm is off the exact solution and does not converge.

For spatial resolution, which was not an issue with the Beer's law test case, as the molecule population was homogeneous, Fig. 7 shows second order convergence by $\mathcal{O}\left(N_{\mathrm{c}}^{2}\right)$ for O'Rourke's algorithm, where $N_{\mathrm{c}}$ is the number of cells, which is compliant with previous publications [20,21, 24]. Fluctuations grow as larger numbers of cells lead to reduced number of parcels per cell, and thus to poor statistics. In the most extreme case, there are no two parcels expected in any cell, and if there are, collision probabilities are huge as the control volume $V$ is small, leading to an erratic response of the collision algorithm. For the hybrid determistic algorithm and Nordin's hybrid algorithm, spatial resolution cannot be investigated, as these algorithms do not involve any control volumes by concept.

The influence of the number of parcels $N_{\mathrm{p}}$ is found to be in strong agreement with previous publications [20, 21, 24].

For the O'Rourke algorithm, the collision rate converges by $\mathcal{O}\left(N_{\mathrm{p}}^{1 / 2}\right)$, Fig. 8, which is expected behavior. The mean error of the weighted deterministic algorithm is still governed by the finite size of the parcel population, i.e. by parcels 

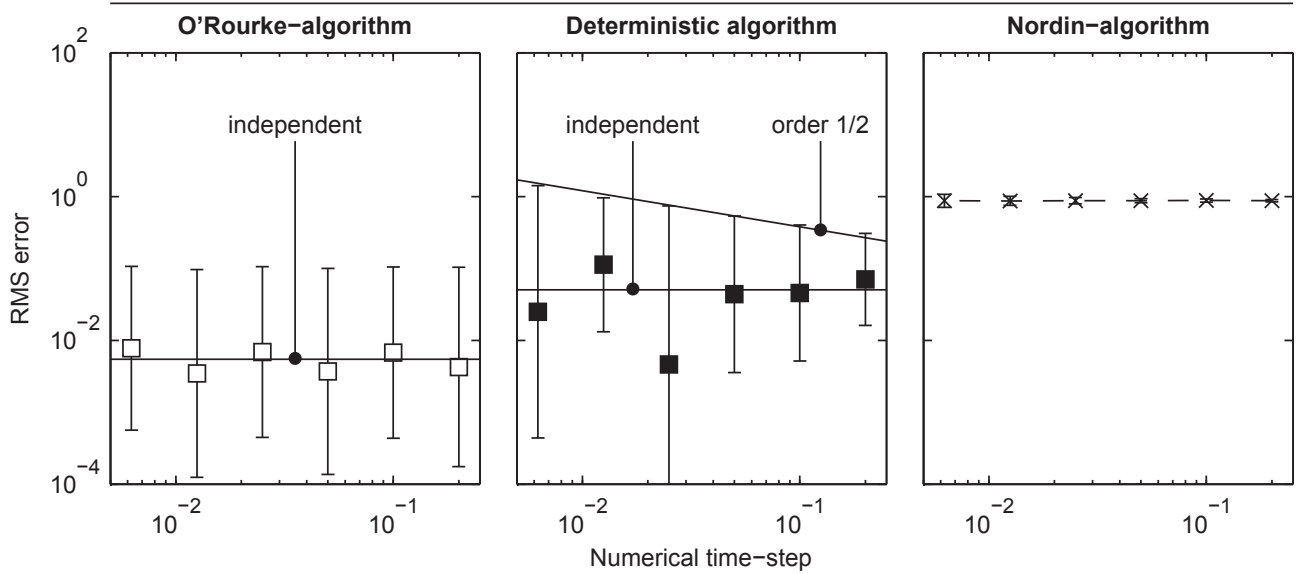

Figure 6: Response of the collision rate test case to variable time-steps. The mean error of the O'Rourke- and weighted deterministic algorithm are independent of time step; the variance of the weighted deterministic algorithm converges by $\mathcal{O}\left(\Delta t^{1 / 2}\right)$. Nordin's hybrid algorithm does not show convergence, but rather a persistently large error.

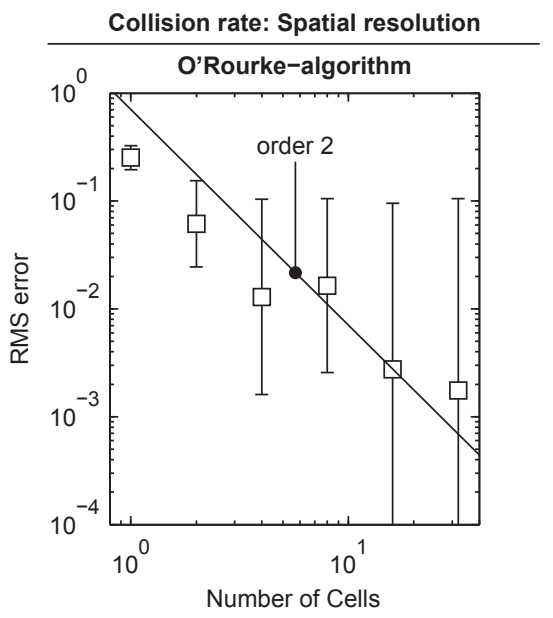

Figure 7: Response of the collision rate test case to variable numbers of cells. The O'Rourke-algorithm converges by $\mathcal{O}\left(N_{\mathrm{c}}^{2}\right)$ as known from other publications [24]. The other algorithms do not make use of any collision mesh.

leaving the parcel populated area. Standard deviations decrease by $\mathcal{O}\left(N_{\mathrm{p}}^{1 / 2}\right)$ as additional parcels increase the number of collisions and thus improve statistics. Both algorithms do not respond to changes in reference frame velocity, as shown in Fig. 9. Nordin's hybrid algorithm does not converge to the exact solution and responds very sensitively to the reference frame velocity with errors of several orders of magnitude.

Changes in the particle diameter do not affect the correctness of O'Rourke's algorithm and the weighted deterministic algorithm. However, larger diameters lead to more collisions and thus improve statistics, visible by a reduction of standard deviations by $\mathcal{O}\left(d^{1 / 2}\right)$ in Fig. 10. The error of Nordin's hybrid algorithm responds sensitively to the drop diameter, which is due to the inproper scaling with the collision cross-section in (14). 


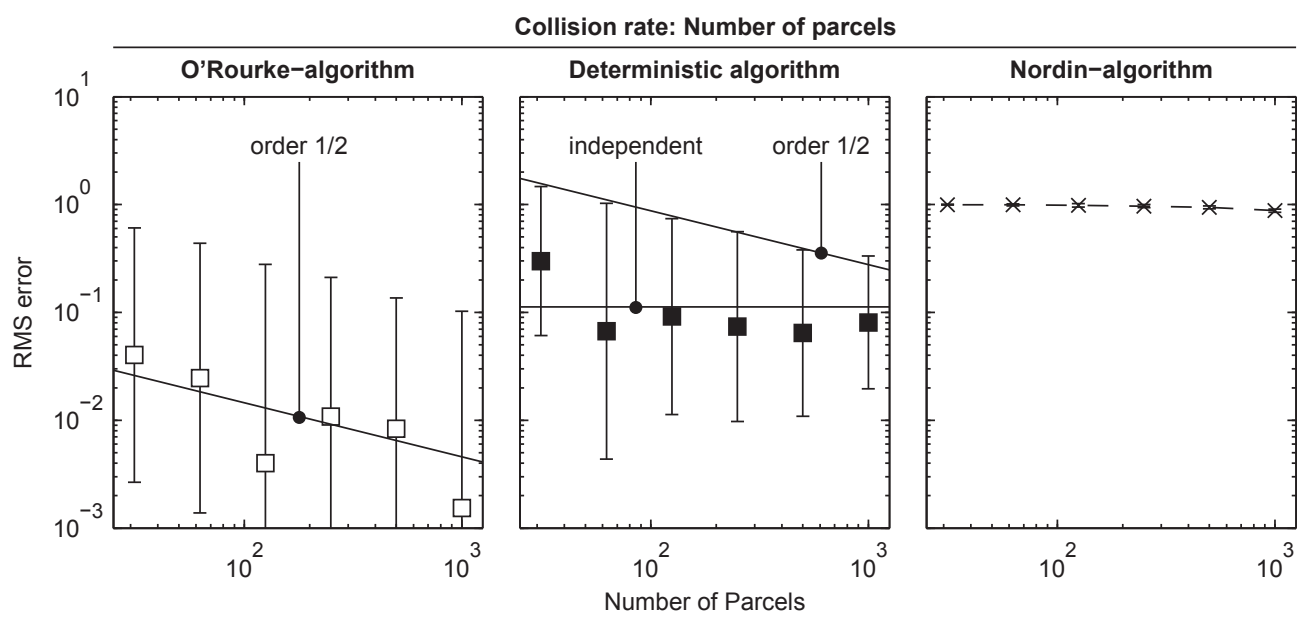

Figure 8: Response of the collision rate test case to variable numbers of parcels. The mean error of the O'Rourkealgorithm by $\mathcal{O}\left(N p^{1 / 2}\right)$ as known from other publications [24]. The mean error of the deterministic algorithm is independent of the number of parcels, with variances decreasing by $\mathcal{O}\left(N p^{1 / 2}\right)$ for larger sample sizes. Nordin's hybrid algorithm does not converge.

\section{Conclusions}

Three collision algorithms were tested in the course of this work: a weighted deterministic algorithm, O'Rourke's stochastic algorithm [17], and a hybrid algorithm proposed by Nordin [15]. We chose these three algorithms because they each have a special significance in the field of particle colllision modeling.

O'Rourke's algorithm was chosen because it represents a seminal work in collision calculations. Though it has been subject to criticism in the open literature, it is very widely used, and is standard in commercial CFD codes such as ANSYS Fluent. The algorithm's derivation stems from basic probability and is stochastic.

Nordins's hybrid algorithm was chosen because it purports to be an improvement on O'Rourke's method and has gained some popularity in industry and academia. At first glance, the hybrid collision algorithm appears reasonable, since it scales qualitatively with relative velocities and particle-sizes. However, further analysis of the algorithm has revealed serious mathematical inconsistencies, which prohibit the use of the algorithm in scientific simulations.

The weighted deterministic algorithm shows how one might mix deterministic with stochastic modeling in a more consistent fashion. The incidence of collision depends on geometric considerations of particle paths instead of probablistic tests. This geometric basis addresses past concerns that O'Rourke's algorithm does not treat the location and trajectory of parcels with sufficient fidelity.

Candidate algorithms were examined using both analytical and empirical testing. The results show that both the weighted deterministic and stochastic algorithms were of comparable quality; each produced converging errors and reference frame invariance. The stochastic nature of the algorithms presents some statistical scatter in the results, without sacrificing overall accuracy.

The advantage of stochastic algorithms is higher computational efficiency, as parcels may be presorted in control volumes which can be handled efficiently and parallelly. Stochastic algorithms are the method of choice for wall bounded disperse flows, as long as particle number densities are relatively homogeneous. The advantage of weighted deterministic 


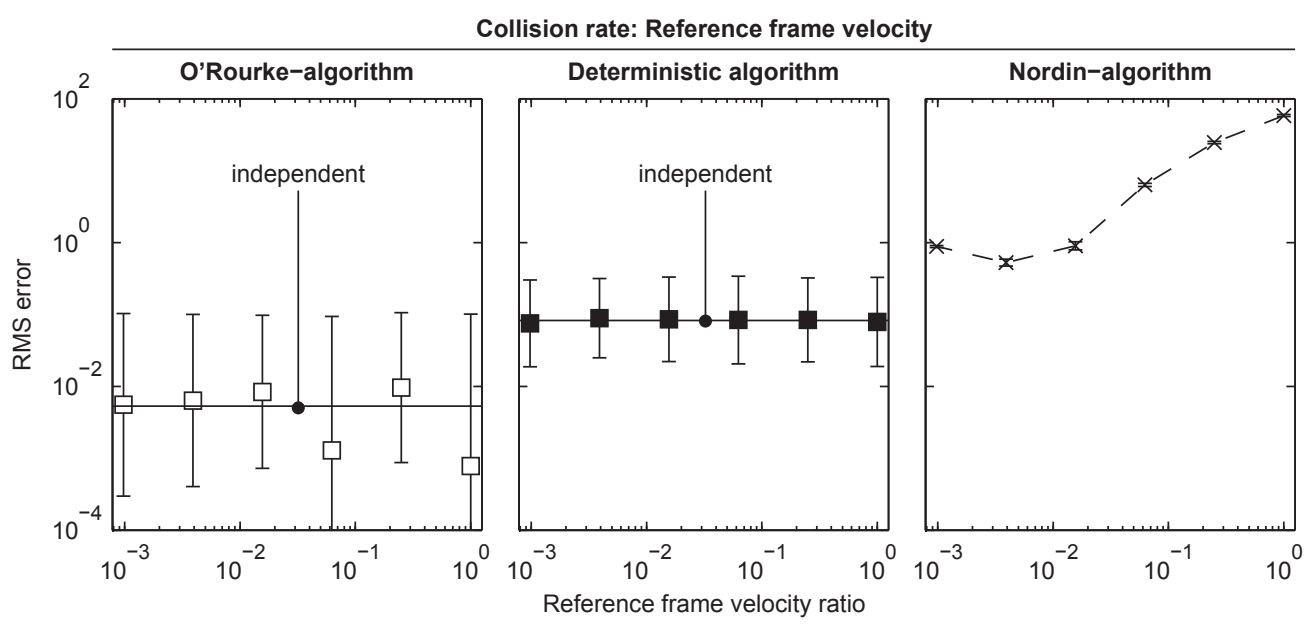

Figure 9: Response of the collision rate test case to variable reference frame velocity ratios (ref. frame velocity/relative velocity). The errors of the O'Rourke- and weighted deterministic algorithms are independent of reference frame; Nordin's hybrid algorithm diverges into both directions.

algorithms is the lack of any control volumes, and thus any control volume dependencies. The price to be paid is lower computational efficiency, and issues with wall bounded flows if the weighted collision cross-sections grow out of the fluid domain, which is not an issue with stochastic algorithms if the collision mesh is aligned with the domain boundaries.

The similar accuracy of stochastic and deterministic algorithms indicates that deterministic logic is no prerequisite to accurate stochastic collision predictions. For example, there is no need to check if parcels approach or not, as exact particle positions are unknown in Monte Carlo simulations anyway. One could imagine changing the deterministic algorithm to backward discretization (predicting collisions in the previous time-step instead of the future time step), which would restrict collisions to parcels that have passed and moved away from each other - without changing the result.

For Nordin's hybrid algorithm, mathematical analysis indicates that the results cannot be correct, as the algorithm contains several fundamental flaws. These flaws were confirmed with numerical tests, where the hybrid algorithm resulted in errors that were orders of magnitude higher than the other two methods. In all cases, the Hybrid Algorithm was found not to be convergent at all. This is particularly concerning as Nordin's algorithm has been incorporated into the OpenFOAM $^{\circledR}$ and AVL Fire ${ }^{\circledR}$ CFD codes and is widely used in spray simulations.

The larger concern becomes how to ensure that new algorithms provide reliable predictions of collision incidence. The appeal of collision algorithms is that contrary to physical models, algorithms can be put to analytical tests and numerical validation. The following is a list of a-priori checks to test prospective collision algorithms prior to any validation:

- Reasonability: The algorithm must not contain any non-physical formulations. Questionable assuptions must be tested rigorously.

- Generelizability: The algorithm must deliver a response for all combinations of parcel properties, such as statistical weight $q$, diameters $d$, velocities $u$ etc.

- Scalability: The number of collisions must scale with particle number density, relative velocity, collision crosssection, and numerical time-step. For turbulence-based collision algorithms not considered here, other quantities may be influential. 


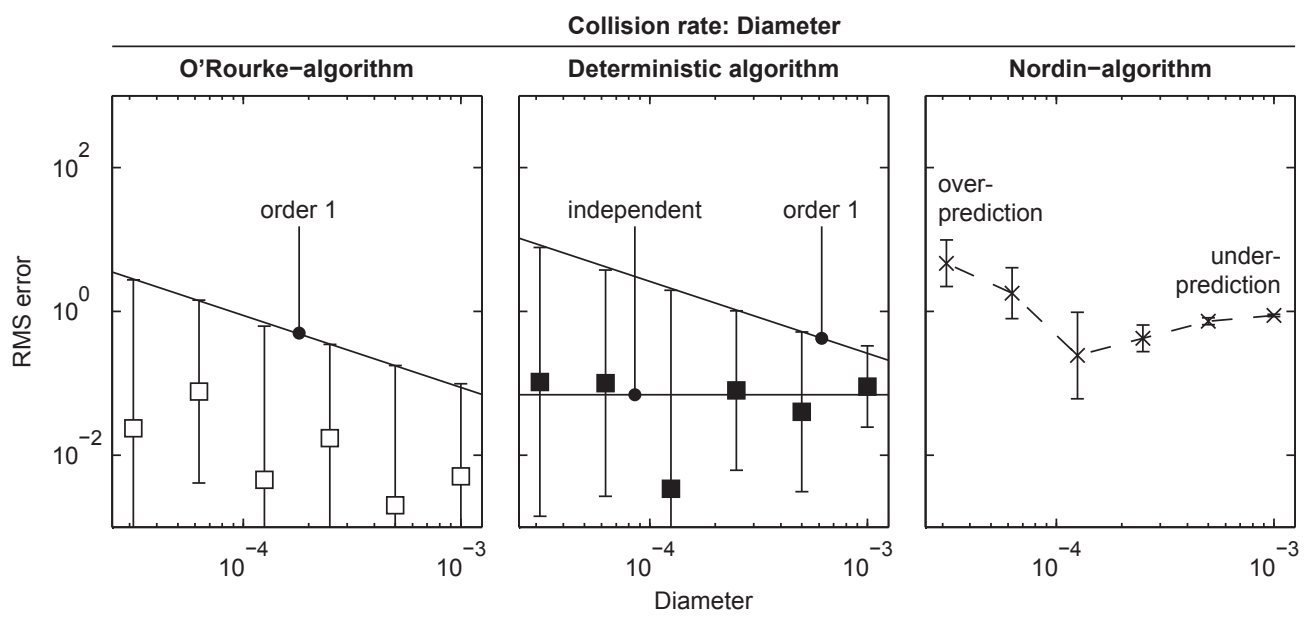

Figure 10: Response of the collision rate test case to variable particle diameters. The mean error of the O'Rourkeand weighted deterministic algorithms is independent of diameter, with $\mathcal{O}(d)$ decreasing statistical scatter for larger diameters and larger numbers of collisions; Nordin's hybrid algorithm diverges into both directions due to incorrect scaling with the collision cross section.

- Frame invariance: To be frame invariant, collision probabilities and deterministic collision criteria must be formed strictly on relative velocities only.

- Numerical invariance: Except for normal convergence behavior, the algorithm must be invariant to numerical parameters.

Both the weighted deterministic algorithm and O'Rourke's algorithms pass the tests above, where the hybrid algorithm fails. After testing, the algorithms must be put to rigorous validation against simplified test cases, which can be compared either to direct calculations $[20,21]$ or analytical solutions [24]. The use of collision algorithms without testing and validation is ill-advised, even if the predictions appear resonable at first glance.

\section{Conflict of Interest}

The authors of this study have presented own collision algorithms before [9, 20,21, 24, 25]. The algorithms were subject to validation and published in peer-reviewed journals. In this study, these algorithms have not been tested to avoid any bias in the conclusions.

\section{Acknowledgments}

The authors thank the German Research Foundation for funding the SFB 686 joint research project "Model-Based Control of Homogenized Low-Temperature Combustion".

\section{References}

[1] Abani, N., Munnannur, A., Reitz, R.D., 2008. Reduction of numerical parameter dependencies in diesel spray models. J. Eng. Gas Turbines Power 130, 032809. 
[2] Ashgriz, N., Poo, J.Y., 1990. Coalescence and separation in binary collisions of liquid drops. J. Fluid Mech. 221 (1), $183-204$.

[3] AVL, 2013. AVL FIRE ${ }^{\circledR}$ Lagrangian multiphase. Tech. Rep. Doc.No: 04-01-05010, Advanced Simulation Technologies AVL List GmbH.

[4] Brenn, G., Valkovska, D., Danov, K.D., 2001. The formation of satellite droplets by unstable binary drop collisions. Phys. Fluids $13,2463$.

[5] Chen, M., Kontomaris, K., McLaughlin, J.B., 1999. Direct numerical simulation of droplet collisions in a turbulent channel flow. part i: collision algorithm. Int. J. Multiphase Flow 24 (7), 1079-1103.

[6] Estrade, J.-P., Carentz, H., Lavergne, G., Biscos, Y., 1999. Experimental investigation of dynamic binary collision of ethanol droplets - a model for droplet coalescence and bouncing. Int. J. Heat Fluid Flow 20 (5), 486-491.

[7] Gustavsson, J., Golovitchev, V., 2004. 3D simulation of multiple injections in DI diesel engines. In: International Symposium on Diagnostics and Modeling of Combustion in Internal Combustion Engines.

[8] Gwon, H.K., Ryou, H.S., 2005. Droplet collision processes in an inter-spray impingement system. J. Aerosol Sci. 36 (11), $1300-1321$.

[9] Hou, S., Are, S., Schmidt, D.P., 2003. A generalized adaptive collision mesh for multiple injector orifices. In: SAE World Congress 2003.

[10] Hou, S., Schmidt, D.P., 2006. Adaptive collision meshing and satellite droplet formation in spray simulations. Int. J. Multiphase Flow 32, 935-956.

[11] Kollár, L.E., Farzaneh, M., Karev, A.R., 2005. Modeling droplet collision and coalescence in an icing wind tunnel and the influence of these processes on droplet size distribution. Int. J. Multiphase Flow 31 (1), $69-92$.

[12] MacInness, J.M., Bracco, F.V., 1991. Comparisons of deterministic and stochastic computations of drop collisions in dense sprays. Prog. Astronautics and Aeronautics 135, 615-642.

[13] Munnannur, A., Reitz, R.D., 2008. Comprehensive collision model for multidimensional engine spray computations. Atomization and Sprays 19, 597-619.

[14] Nobari, M.R.H., Tryggvason, G., 1996. Numerical simulations of three-dimensional drop collisions. AIAA journal 34 (4), $750-755$.

[15] Nordin, N., 2001. Complex chemistry modeling of diesel spray combustion. Ph.D. thesis, Chalmers University of Technology.

[16] Orme, M., 1997. Experiments on droplet collisions, bounce, coalescence and disruption. Prog. Energy Comb. Sci. $23,65-79$.

[17] O'Rourke, P.J., 1981. Collective drop effects on vaporizing sprays. Ph.D. thesis, Department of Mechanical and Aerospace Engineering, Princeton University.

[18] Pan, Y., Suga, K., 2005. Numerical simulation of binary liquid droplet collision. Phys. Fluids 17, paper 082105.

[19] Pawar, S.K., Padding, J.T., Deen, N.G., Kuipers, J.A.M., Jongsma, A., Innings, F., 2012. Eulerian-lagrangian modelling with stochastic approach for droplet-droplet collisions. In: Ninth International Conference on CFD in the Minerals and Process Industries. CSIRO.

[20] Pischke, P., Cordes, D., Kneer, R., 2012. A collision algorithm for anisotropic disperse flows based on ellipsoidal parcel representations. Int. J. Multiphase Flow 38, 1-16.

[21] Pischke, P., Cordes, D., Kneer, R., 2012. The velocity decomposition method for second-order accuracy in stochastic parcel simulations. Int. J. Multiphase Flow 47, 160-170.

[22] Post, S.L., Abraham, J., 2002. Modeling the outcome of drop-drop collisions in Diesel sprays. Int. J. Multiphase Flow 28 (6), $997-1019$.

[23] Qian, J., Law, C.K., 1997. Regimes of coalescence and separation in droplet collision. J. Fluid Mech. 331, 59-80.

[24] Schmidt, D.P., Rutland, C.J., 2000. A new droplet collision algorithm. J. Comp. Phys. 164, 62-80.

[25] Schmidt, D.P., Rutland, C.J., 2004. Reducing grid dependency in droplet collision modeling. J. Eng. Gas Turbines Power 126, $227-233$.

[26] Sommerfeld, M., 2001. Validation of a stochastic lagrangian modelling approach for inter-particle collisions in homogeneous isotropic turbulence. Int. J. Multiphase Flow 27 (10), 1829-1858.

[27] Subramaniam, S., 2012. Lagrangian-Eulerian methods for multiphase flows. Prog. Energy Comb. Sci. 39, $215-245$.

[28] Wang, L.P., Wexler, A.S., Zhou, Y., 2000. Statistical mechanical description and modeling of turbulent collision of inertial particles. J. Fluid Mech. 415, 117-153.

[29] Wang, L.-P., Ayala, O., Kasprzak, S.E., Grabowski, W.W., 2005. Theoretical formulation of collision rate and collision efficiency of hydrodynamically interacting cloud droplets in turbulent atmosphere. J. Atmosph. Sci. 62 (7), 2433-2450.

[30] Weller, Henry G., G.Tabor, Jasak, Hrvoje, Fureby, C., 1998. A Tensorial Approach to Computational Continuum Mechanics Using Object-Oriented Techniques. Computers in Physics 12 (6), 620-631. 